\title{
Dark Photon Searches Using Displaced Vertices at Low Energy $e^{+} e^{-}$Colliders
}

\author{
Fabio Bossi \\ Laboratori Nazionali dell'INFN, Via E. Fermi 40, 00044 Frascati, Rome, Italy \\ Correspondence should be addressed to Fabio Bossi; fabio.bossi@lnf.infn.it
}

Received 26 November 2013; Accepted 29 January 2014; Published 11 March 2014

Academic Editor: Jean-René Cudell

Copyright (c) 2014 Fabio Bossi. This is an open access article distributed under the Creative Commons Attribution License, which permits unrestricted use, distribution, and reproduction in any medium, provided the original work is properly cited. The publication of this article was funded by SCOAP .

\begin{abstract}
The existence of a new, photon-like, massive particle, the $\gamma^{\prime}$ or dark photon, is postulated in several extensions of the Standard Model. These models are often advocated to explain some recent puzzling astrophysical observations, as well as to solve the so far unexplained deviation between the measured and calculated values of the muon anomaly. Dark photons can be produced at $e^{+} e^{-}$ colliders both in continuum events and in vector meson transitions and can eventually decay into an electron-positron pair. For a proper choice of the parameters of the theory, a $\gamma^{\prime}$ can have a relatively long lifetime and can therefore be observed as an $e^{+} e^{-}$ vertex well separated by the primary interaction point. This case is discussed in reference to very high luminosity $e^{+} e^{-}$colliders either in construction or under study in several laboratories in the world. It is shown that a search strategy based on the detection of displaced vertices can be in principle very effective in covering a rather wide and to date unexplored region of the theoretical parameters space.
\end{abstract}

\section{Introduction}

In the Standard Model (SM), interactions among elementary particles are mediated by the vector bosons of the strong, weak, and electromagnetic forces. Experimental evidence for the existence of those bosons is compelling and precise measurements of their properties have been accumulated in the past decades. New forces can have escaped detection so far, either if their associated bosons are very heavy or if their couplings to ordinary matter are weak enough. The latter case has been advocated, among others, in models which try to explain and reconcile among them several puzzling astrophysical observations performed in recent years [1-4]. They are sometimes also used to reconcile the measured value of the muon anomaly to the SM prediction, which differ by approximately by $3.5 \sigma$ (see, e.g., [5]).

If new, light, neutral bosons (which from now on will be called $\gamma^{\prime}$ or dark photons) exist and if they are measurably, albeit weakly, coupled with SM particles, they can be produced and observed at colliding-beams and fixed target experiments [6-12]. In fact, there have been several attempts to observe evidence for such particles, using data from running facilities [13-19] or data mining old experiments $[7,20-23]$. Since no evidence for their existence was found, limits have been set as a function of the $\gamma^{\prime}$ mass and of its coupling to ordinary matter.

In the near future, new experiments under construction are expected to extend those limits in a region of couplings and/or masses so far unexplored. All of them are designed to exploit the radiative production of the $\gamma^{\prime}$ by a very intense electron or positron beam on a properly built high $-Z$ target [24-29]. The purpose of the present letter is to show that comparable results can be obtained by high luminosity and low energy electron-positron colliders, such as those under construction or under study in several laboratories in the world [30-32]. These facilities will take advantage of two main construction features which coherently conspire to enhance their discovery potential: their very high goal luminosity and the usage of very compact beams (these two features are in fact strongly correlated). Actually, high luminosity translates into the possibility of probing lower production cross sections, that is, lower effective couplings between the $\gamma^{\prime}$ and ordinary matter. On the other hand, low couplings translate into longer $\gamma^{\prime}$ decay paths, especially for low $\gamma^{\prime}$ 
masses. Thus, the usage of beams of very small dimensions allows one to obtain a clear $\gamma^{\prime}$ signal by observing secondary vertices of a well-defined invariant mass, well separated by the beams interaction point.

In the paper, this case will be discussed for three different possible choices of the machine center-of-mass energy, corresponding, respectively, to the mass of the $\phi(1020)$, the $J / \psi(1 S)$, and the $\Upsilon(4 S)$ mesons. This choice is motivated by the projects mentioned above. It will be shown that higher energy machines are favoured, not only because they are expected to deliver larger data sets, but also because the $\gamma^{\prime}$ therein produced have longer decay paths, ceteris paribus. Instrumental effects play however a relevant role in the actual detection strategy and can in some cases dramatically reduce the discovery potential of the method. Still, in particular for the case of a high luminosity $\tau$-charm factory, it remains high enough to be competitive to the fixed target experiments mentioned above.

The paper is organized as follows. Firstly, the theoretical framework of the paper is discussed, together with a short presentation of the experimental limits on the existence of dark photons obtained so far. The case for the searches at low energy, high luminosity $e^{+} e^{-}$colliders is discussed in Section 3, followed by some considerations on the actual implementation of the proposed method to existing, or planned, facilities. Radiative vector meson decays are discussed in Section 5. Conclusions are given in Section 6.

\section{Physics Case}

In many new physics scenarios, the $\mathrm{SM}$ is extended by simply adding an additional $U(1)_{D}$ symmetry, under which SM particles are uncharged at first order $[5,33,34]$. The gauge boson associated to the new symmetry, the $\gamma^{\prime}$, can still interact with ordinary matter via kinetic mixing described by an effective interaction Lagrangian. Consider

$$
L_{\text {kin-mix }}=i \epsilon e \bar{\psi}_{\mathrm{SM}} \gamma^{\mu} \psi_{\mathrm{SM}} A_{\mu} \text {, }
$$

where $A$ denotes the $\gamma^{\prime}$ field. The kinetic mixing factor $\epsilon$ parametrizes the coupling strength relative to the electric charge and is predicted in various models to be in the range $10^{-12}-10^{-2}$. The mass of the dark photon $\left(m_{\gamma^{\prime}}\right)$ rests unpredicted. On phenomenological grounds, however, masses in the $\mathrm{MeV}-\mathrm{GeV}$ range, which are of interest for the present work, are favoured.

There might exist non-SM matter particles which are sensitive to the new $U(1)_{D}$ interaction. Often they are postulated to be the main constituent of the yet undiscovered dark matter component of the universe (DM) and must therefore be electrically neutral and stable. If kinematically allowed, the $\gamma^{\prime}$ will decay preferably into pairs of these particles; thus its decay becomes "invisible." The case for detecting invisible decays is discussed, for instance, in [28,35-38]. On the other hand, if the dark photon is lighter than DM, it is forced to

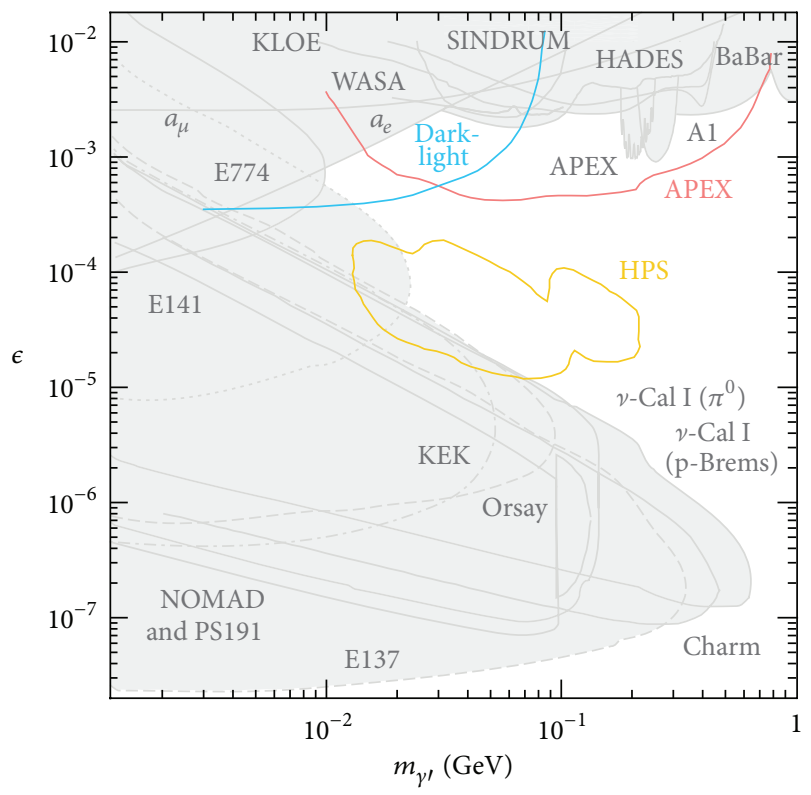

FIGURE 1: Excluded region in the plane $\epsilon-m_{\gamma^{\prime}}$ resulting from presently available data. Electron beam dump experiment covers the region of low masses and very low couplings, down to $\epsilon \sim 10^{-7}$. For higher masses and lower couplings limits come mainly from meson decays and electron-nucleon scattering experiments and to B-factories data (plot courtesy of S. Andreas). The plot reports also the projections for the experiments presently under construction. For details on single experiments see [39].

decay into a pair of SM particles, with a width regulated by (1). In this case, its proper time is approximately given by [39]

$$
c \tau(\mathrm{mm})=\frac{0.08}{N_{f}} \cdot\left(\frac{10^{-4}}{\epsilon}\right)^{2} \cdot \frac{100}{m_{\gamma^{\prime}}(\mathrm{MeV})},
$$

where $N_{f}$ is the number of SM decay channels allowed by kinematics.

There have been several attempts to experimentally observe a $\gamma^{\prime}$ signal, using many different techniques. Figure 1, taken from reference [39], shows the exclusion plot in the plane $m_{\gamma^{\prime}}-\epsilon$, resulting from the above mentioned searches. Electron beam dump experiment covers the region of low masses and very low couplings, down to $\epsilon \sim 10^{-7}$. For higher masses and lower couplings, limits come mainly from meson decays and electron-nucleon scattering experiments and to Bfactories data. Important information, not shown in Figure 1, can also be deduced by astrophysical observations (see for instance [40] and references therein). For $m_{\gamma^{\prime}}>10-20 \mathrm{MeV}$, the region with $\epsilon<10^{-3}$ remains largely unexplored.

There are currently various experiments either running or under construction aiming at probing part of this region. All of them are designed to exploit the radiative production of the $\gamma^{\prime}$ by a very intense electron or positron beam on a properly built high- $Z$ target. In particular, the HPS experiment at Thomas Jefferson Laboratory (USA) is designed to cover the region $\epsilon=\left(10^{-4}, 10^{-5}\right), m_{\gamma^{\prime}}=(20,300) \mathrm{MeV}$ [25] (see Figure 1) 
In the following, the case for the search for dark photons in the same parameters space region at a very high luminosity $e^{+} e^{-}$collider will be discussed.

\section{Searches at $e^{+} e^{-}$Colliders}

In the last decades, large amount of data have been collected at high luminosity $e^{+} e^{-}$flavour factories operating at different center of mass energies. These data range from the $\sim 2 \mathrm{fb}^{-1}$ delivered at the $\phi(1020)$ peak by the Italian collider DAФNE to the $0.5-1 \mathrm{ab}^{-1}$ produced by the B-factories at PEP-II (USA) and KEK-B (Japan). In the near future, a consistent increase of the above statistics is expected both at DAФNE and at KEK-B which aims at increasing their data sample by factors of 10 and 50, respectively. An option to increase the center of mass energy of DAФNE up to $2.5 \mathrm{GeV}$ has been taken into consideration [41]. Finally, studies for the construction of a collider capable of delivering $\sim 1 \mathrm{ab}^{-1}$ around the charm threshold are under consideration in Italy, Russia, and China (see for instance [31]).

As of today, searches for dark photons at $e^{+} e^{-}$colliders have been pursued mainly by studying the process $e^{+} e^{-} \rightarrow$ $\gamma \gamma^{\prime}$ with the subsequent decay of the $\gamma^{\prime}$ into a $\mu^{+} \mu^{-}$pair. This limits the search to $m_{\gamma^{\prime}}>2 m_{\mu}$, which, as a consequence of (2), results in its lifetime being unmeasurably short. Therefore, the signal can be separated by the more copious and otherwise indistinguishable QED background, only by observing a sharp peak in the invariant mass distribution of the final state lepton pair.

The question arises whether it would be possible to extend the search also to the region with $m_{\gamma^{\prime}}<2 m_{\mu}$ and in particular with $\epsilon<10^{-3}$. The main message of the present paper is that the foreseen increase of the potentially available data sample allows one to give a positive answer to the above question, not only because of the increased statistical sensitivity but also because it opens the doors to the possibility of observing a clear signal for a long-lived $\gamma^{\prime}$, which is only marginal with the presently available data.

Here and in what follows, for the sake of simplicity, only the case of symmetric machines is considered. Also, since we are interested in the case with $m_{\gamma^{\prime}}<2 m_{\mu}$, the dark photon can decay only into a $e^{+} e^{-}$pair.

The differential cross section for radiative $\gamma^{\prime}$ production in $e^{+} e^{-}$collisions is given by [11]

$$
\frac{d \sigma}{d \cos \theta}=\frac{2 \pi \alpha^{2} \epsilon^{2}}{E_{\text {c.m. }}^{2}} \cdot \frac{1+\cos ^{2} \theta}{\sin ^{2} \theta},
$$

where $\theta$ is the angle between the incoming positron and the outcoming photon and $E_{\text {c.m. }}$ denotes the center of mass energy of the event. By integrating the above equation between $\cos (\theta)= \pm 0.8$, one obtains a total cross section of approximately $0.9 \times \epsilon^{2} \mu \mathrm{b}, 0.1 \times \epsilon^{2} \mu \mathrm{b}$, and $3.6 \times \epsilon^{2} \mathrm{nb}$ for $E_{\text {c.m. }}=1.02,3.1$, and $10.5 \mathrm{GeV}$, respectively. Thus, in the presently available data samples, for $\epsilon=10^{-4}$ a few dozens $\gamma^{\prime}$ can be found.

Due to the two body kinematics, the dark photon is boosted in the laboratory frame by a factor $E_{\text {c.m. }} . m_{\gamma^{\prime}}$. Therefore, for small kinetic mixings and for low enough dark photon masses, its lifetime in the laboratory frame becomes sizeable. For instance, for $\epsilon=10^{-4}$ and $m_{\gamma^{\prime}}=20 \mathrm{MeV}$, the mean decay path of a dark photon is $\sim 1,3$, and $10 \mathrm{~cm}$ for $E_{\text {c.m. }}=1.02,3.1$, and $10.5 \mathrm{GeV}$, respectively.

Can these long decay paths be exploited to separate a potential $\gamma^{\prime}$ signal from the QED background? Clearly, although the secondary vertex can be determined with standard vertexing techniques, cannot be, on an event by event basis. On the other hand, the actual position and size of the collision envelope can be determined on a statistical basis using known processes, such as Bhabha scattering or muon pair production. Interestingly, at all of the $e^{+} e^{-}$facilities under consideration, one of the strategies used to maximize luminosity is to keep the transverse beam dimensions at the interaction point as small as possible, typically $\leq 1 \mathrm{~mm}$. Therefore, assuming a perfectly Gaussian distribution of the beam spot, with a maximum transverse dimension of $1 \mathrm{~mm}$, the probability for observing an $e^{+} e^{-}$vertex from standard QED processes at a transverse distance of $1 \mathrm{~cm}$ or more from the center of the collision spot is practically zero. On the other hand, the number of $\gamma^{\prime}$ decay events with transverse decay path larger than $1 \mathrm{~cm}, N_{1 \mathrm{~cm}}$ can be as large as several thousands, depending on the actual value of $\epsilon, m_{\gamma^{\prime}}$, and $E_{\text {c.m. }}$. and of the luminosity integrated by the machine, $L_{\text {int }}$.

Figure 2 shows the variation of $N_{1 \mathrm{~cm}}$ as a function of $\epsilon$, for different values of $m_{\gamma^{\prime}}$ and for three different experimental conditions: (a) $E_{\text {c.m. }}=1.02 \mathrm{GeV}, L_{\text {int }}=20 \mathrm{fb}^{-1}$; (b) $E_{\text {c.m. }}=$ $3.1 \mathrm{GeV}, L_{\text {int }}=1 \mathrm{ab}^{-1}$; (c) $E_{\text {c.m. }}=10.5 \mathrm{GeV}, L_{\text {int }}=$ $50 \mathrm{ab}^{-1}$. The chosen values for $L_{\text {int }}$ correspond to the target performance for the facilities under construction or under study mentioned above. The behaviour of the curves is easily explained. For $\epsilon \ll 10^{-4}$ the mean decay path of a dark photon is much larger than $1 \mathrm{~cm}$, and therefore $N_{1 \mathrm{~cm}}$ increases with $\epsilon^{2}$, independently of $m_{\gamma^{\prime}}$. It eventually reaches a peak and drops rapidly towards zero, as long as, with increasing $\epsilon$, the lifetime becomes shorter and shorter. The position of the peak is determined by the proper balance between the effect of the production cross section, which increases with $\epsilon^{2}$, and that of the lifetime which decreases with it. It depends also on the value of $m_{\gamma^{\prime}}$, the decay path decreasing again quadratically with it. It is seen that, despite the lower production cross section, the largest expected integrated luminosity combined with the higher boost factors favours the B-factory option (case (c)). In this case, however, the peak of the distribution, especially for lower masses, is obtained for values of the kinetic mixing $\sim 10^{-3}$. It can be noted also that in case (a) the number of observable dark photons with masses greater than $\sim 30 \mathrm{MeV}$ becomes hopelessly small. This is not only due to the lower luminosity but also to the reduced Lorentz boost, consequence of the lower center of mass energy of the collision.

Although the results obtained so far look very encouraging on general grounds, there are two main limitations coming from the implementation of the above search strategy into a real experiment. On the one hand, for specific values of the parameters, the $\gamma^{\prime}$ lifetime becomes so long that a relevant part of the decays would escape detection of an apparatus of realistic dimensions. For instance, 


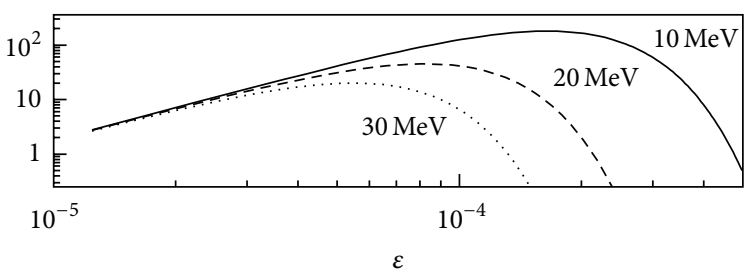

(a)

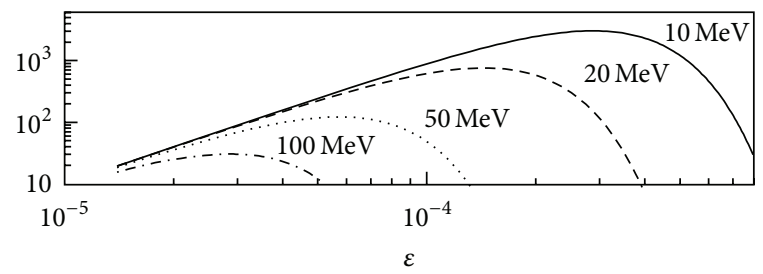

(b)

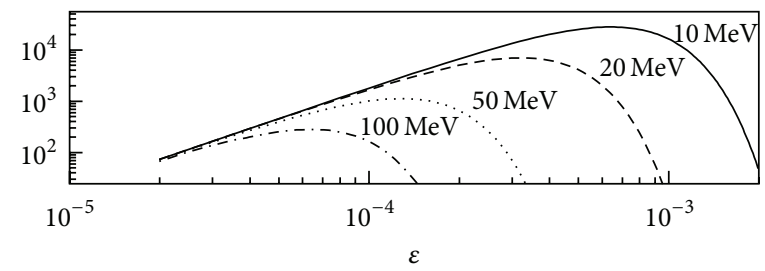

(c)

FIGURE 2: Number of $\gamma^{\prime}$ decay events with transverse decay path larger than $1 \mathrm{~cm}$, as a function of $\epsilon$, for different values of $m \gamma^{\prime}$. Three different experimental conditions are taken into consideration: (a) $E_{\text {c.m. }}=1.02 \mathrm{GeV}, L_{\mathrm{int}}=20 \mathrm{fb}{ }^{-1} ;(\mathrm{b}) E_{\mathrm{c} . \mathrm{m} .}=3.1 \mathrm{GeV}, L_{\mathrm{int}}=1 \mathrm{ab}{ }^{-1} ;(\mathrm{c}) E_{\mathrm{c} . \mathrm{m} .}=$ $10.5 \mathrm{GeV}, L_{\text {int }}=50 \mathrm{ab}^{-1}$. Note that the horizontal scale is slightly different for the three plots.

for $E_{\text {c.m. }}=10.5 \mathrm{GeV}, \epsilon=5 \times 10^{-5}$, and $m_{\gamma^{\prime}}=10 \mathrm{MeV}$, the $\gamma^{\prime}$ mean decay path is about $1.5 \mathrm{~m}$. More importantly, a very dangerous instrumental background comes into operation, namely, photon conversions on the detector material induced by $e^{+} e^{-} \rightarrow \gamma \gamma$ events. This is particularly relevant since experiments are often designed to have beam pipes with very small radii at the interaction point. Although one can try to minimize the conversion probability on the detector elements, by properly choosing type and dimensions of the materials, the cross section of the $e^{+} e^{-} \rightarrow \gamma \gamma$ process is so much larger than the signal one (in fact, it is larger by a factor $\epsilon^{-2}$ ) that this background rapidly becomes unbeatable.

The simplest way to cope with this problem is to allow for a large enough empty region around the interaction point, where photons cannot interact with matter and dark photons can at least partly undergo their decay. It would then be reasonable to accept only events with decay vertices occurring before the beam pipe but still far $(1 \mathrm{~cm})$ from the nominal beam spot center. Assuming a beam pipe of $5 \mathrm{~cm}$ radius, such as that presently used by the KLOE-2 experiment at DAФNE, the number of events thus obtained within acceptance, $n_{\text {acc }}$, is shown in Figure 3 for the three cases under consideration.

For high values of $\epsilon$, this acceptance cut does not observably affect the previous distributions. In fact, in this case, the lifetime is so short that almost all of the dark photons decay much before $5 \mathrm{~cm}$. For lower values of $\epsilon$, instead, the consequence of the cut in acceptance is more visible and can decrease the number of accepted events by an order of magnitude, especially for very low $\gamma^{\prime}$ masses. However, and this is one of the main messages of this paper, the number of potentially observable events remains still considerable for a wide region of the parameter space, especially for the higher energy machine options. In particular, also allowing for some further detection inefficiency, it can be seen that kinetic mixings down to few times $10^{-5}$ and masses up to $\sim 200 \mathrm{MeV}$ can be probed.

Based only on signal statistics (i.e., without taking into consideration possible detector resolution effects and other possible instrumental backgrounds), this translates into the explorable regions shown in Figure 4, for the three cases under consideration. While case (a) covers almost entirely a region already excluded by previous beam-dump experiments, cases (b) and (c) can potentially probe a relatively wide unexplored region (see Figure 1). On the other hand, it has also to be underlined that this same region is expected to be covered by the aforementioned future fixed target experiments (see again Figure 1).

It is worthwhile stressing once more that the requirement for observing a cm-scale decay path ideally rejects every possible physical background to our signal. Still, other instrumental effects need to be taken carefully into account, as discussed in the following section.

\section{Implementation at Current and Future Facilities}

It is of interest understanding how difficult it would be to practically implement on real experimental facilities the ideas discussed so far. This requires a detailed knowledge of the actual machine and detector's design and expected (or measured) performance. Only specific studies based on these figures can in the end determine whether the method is applicable or not, to which extent, and on which machine. An obvious difference between our simplified models and reality can be found for instance in case (c); both the old and the future B-factories are in fact asymmetric machines, the electron beam being of higher energy with respect to the positron one. Although this might somewhat change the specific acceptance requirements with respect to the symmetric 


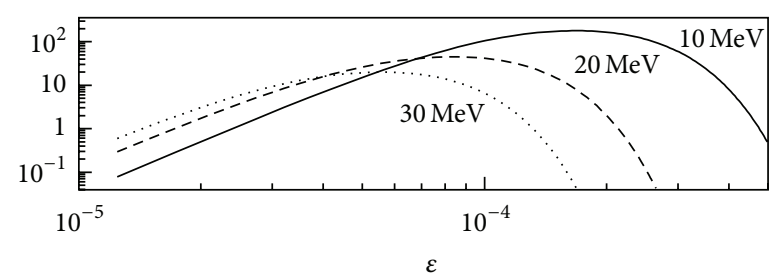

(a)

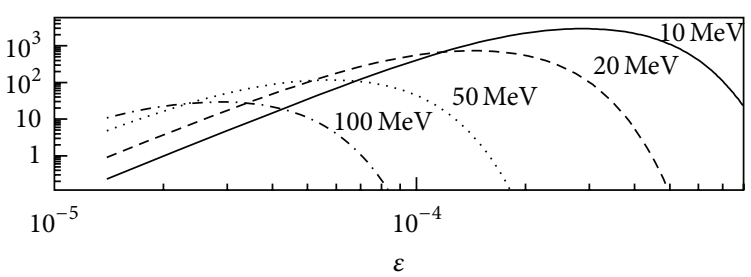

(b)

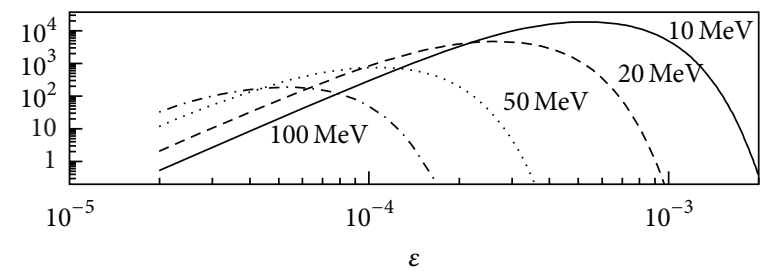

(c)

Figure 3: Same as Figure 2, with the further request that the transverse decay path is lower than $5 \mathrm{~cm}$.

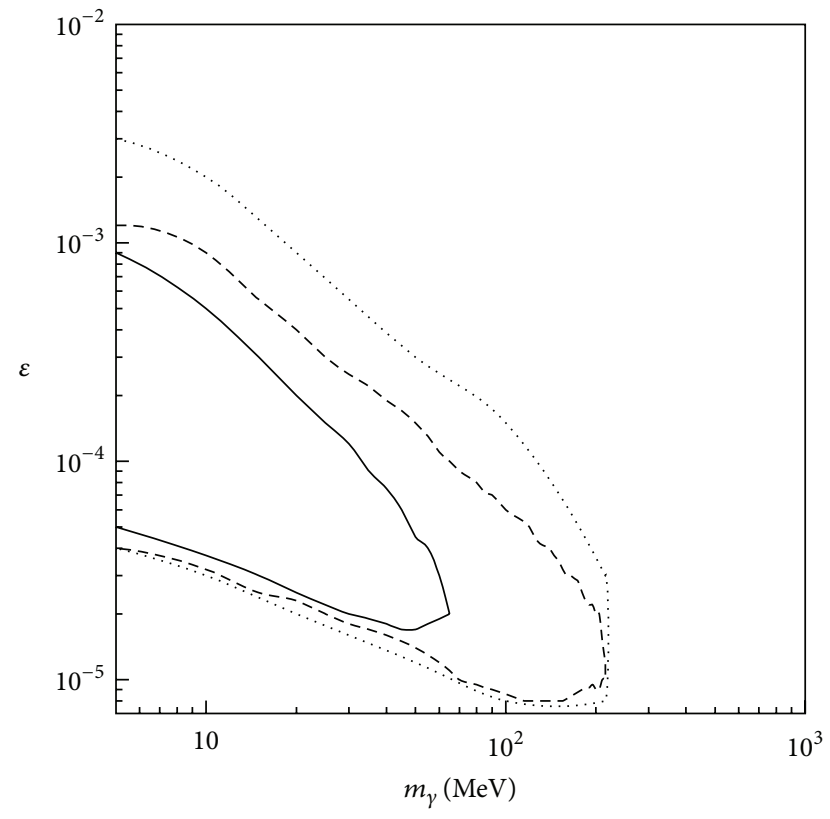

FIGURE 4: Explorable region for cases (a) solid, (b) dashed, and (c) dotted in the plane $\epsilon-m_{\gamma^{\prime}}$. No instrumental backgrounds are taken into consideration, as well as potential efficiency and resolution effects for the detector. Above $\sim 210 \mathrm{MeV}$, the opening of the 2-muon channel drastically reduces the effectiveness of the method.

option discussed in this paper, it is however reasonable to assume that similar conclusions can still be drawn.

On general grounds, there are four parameters that have to be taken into consideration: the primary $\gamma^{\prime}$ production rate, the dimensions of the beams, those of the beam pipe, and the vertexing capabilities of the detector.

As for the first point, despite the higher production cross section, the $\phi$-factory option (case (a)) is less performing than the other two, not only because of the much lower integrated luminosity, but also, as noted before, because of the intrinsic limitation due to the lower Lorentz boost factors. The proposal for running DAФNE at higher energies is, under this respect, particularly interesting because this would allow increasing the decay paths proportionally to $E_{\text {c.m. }}$.

As previously remarked, for all of the machines under consideration, the dimensions of the beams are kept very small in the transverse direction. For instance, at DAФNE, the beams have $\sigma_{X} \sim 1.5 \mathrm{~mm}, \sigma_{y} \sim 0.02 \mathrm{~mm}$, and much lower dimensions at the other machines. Note that both Figures 2 and 3, on which we base our search strategy, refer to transverse decay paths, so the beam dimensions in the longitudinal direction are irrelevant for our conclusions. Obviously, nonGaussian tails of the collision envelope can to some extent increase the background contamination. However, if not completely suppressed, they can still be studied using other known processes, as for the Gaussian part.

A real concern is the actual beam-pipe dimensions. Among the existing facilities, KLOE-2 at DAФNE is the only one having a beam pipe radius at the interaction point of $5 \mathrm{~cm}$. As for the machines running at the charm threshold, the only one presently in operation, the Chinese collider BEPC, has a beam pipe at the interaction point of $3.5 \mathrm{~cm}$ in radius; however, its present luminosity is about a factor 100 lower than that required by our arguments at that energy. For the $\mathrm{B}$-factories, the beam pipe radii range from the $2.5 \mathrm{~cm}$ for the $\mathrm{BaBar}$ detector to $1 \mathrm{~cm}$ for the future SuperBelle.

While a reconsideration of the inner region of SuperBelle is probably very unlikely, in the case of a future high luminosity $\tau$-charm factory, it is conceivable that the interaction region can be designed so as to maximise the sensitivity for the dark photon search under consideration. It is worth noticing here that the request for minimizing the beam pipe dimensions comes to first order from the experiment more than from the machine. In fact, they are somehow anticorrelated with the detector's vertexing capabilities. Actually, the resolution of a decay length measurement for a generic detector is approximately proportional to the single point resolution of the most internal tracking device and inversely proportional to its distance from the decay point. Under 
this respect the less favourable situation is that of KLOE2, whose first tracking device, a triple-GEM cylindrical detector, has an internal radius of $12 \mathrm{~cm}$ and single point resolution of $\sim 200 \mu \mathrm{m}$. Still, its estimated vertex resolution for $K^{0} \rightarrow \pi^{+} \pi^{-}$events is of 1-2 $\mathrm{mm}$ [42]. The use of silicon detectors, which can have single point resolution of order $10 \mu \mathrm{m}$, would definitely improve with respect to the KLOE2 case. Noticeably, all the LEP experiments, which had beam pipes of $5.5 \mathrm{~cm}$, could reach typical decay length resolution of $\sim 250 \mu \mathrm{m}$ for B decay events, thanks to the use of silicon detectors [43]. This implies that, considering the $\mathrm{cm}$ scale decay length we have been interested in so far, vertexing resolution should not be a major issue. On the other hand, it can play a relevant role in the considerations discussed in the following section.

\section{Meson Decays}

Electron-positron colliders provide a useful $\gamma^{\prime}$ production mechanism via radiative vector meson decays, too. Actually, for each observed $V \rightarrow P \gamma$ decay ( $V$ and $P$ being a vector and a pseudoscalar meson, resp.), there could be a $V \rightarrow$ $P \gamma^{\prime}$ process, suppressed by a factor $\epsilon^{2}$ with respect to the former one [9]. This fact has actually been exploited by the KLOE-2 collaboration which has searched for the dark photon using the $\phi \rightarrow \eta e^{+} e^{-}$process in $[17,18]$. As for the searches in the $e^{+} e^{-} \rightarrow \mu^{+} \mu^{-} \gamma$ channel, the signal is separated by the SM (Dalitz decay) background by looking for a peak in the invariant mass distribution of the final state lepton pair. However, once the $\gamma^{\prime}$ lifetime becomes sizeable, these events are characterised as well by the presence of $e^{+} e^{-}$ vertices clearly separated by the collision point, thus allowing the usage of the search strategy described in the previous sections.

The number of produced $\gamma^{\prime}$ is given by

$$
N_{\gamma^{\prime}}=N_{V} \cdot \mathrm{BR}_{V P \gamma} \cdot \epsilon^{2}
$$

where $N_{V}$ is the number of produced vector mesons, and $\mathrm{BR}_{V P \gamma}$ is the branching ratio for the corresponding standard radiative decay.

Let us firstly consider the above mentioned $\phi \rightarrow \eta \gamma^{\prime}$ process. At a $\phi$-factory, $\sim 3 \cdot 10^{9} \phi$ mesons are produced every $\mathrm{fb}^{-1}$ delivered by the machine. Since $\mathrm{BR}_{\phi \rightarrow \eta \gamma} \simeq 1.3 \cdot 10^{-2}$ [44], it is easily seen that the number of produced signal events, assuming $L_{\text {int }}=20 \mathrm{fb}^{-1}$, becomes negligible for $\epsilon \leq 10^{-4}$. On the other hand, for higher values of $\epsilon$, the $\gamma^{\prime}$ mean decay path becomes unmeasurably (as compared with millimeter scale vertex resolutions) short, but for very low masses. For instance, for $\epsilon=2 \cdot 10^{-4}$ it is already $0.8(0.2) \mathrm{cm}$, for $m_{\gamma^{\prime}}=10(20) \mathrm{MeV}$. Unless, therefore, one integrates luminosities largely exceeding those expected from the presently considered machine, the method is hardly applicable to this decay channel.

Let us now turn our attention to the $J / \psi \rightarrow \eta^{\prime} \gamma^{\prime}$ transition. This process has been already studied in [45], where, however, the case for short lived dark photons only is considered. As before, one has $N_{J / \psi} \simeq 3 \cdot 10^{9} / \mathrm{fb}^{-1}$, running at

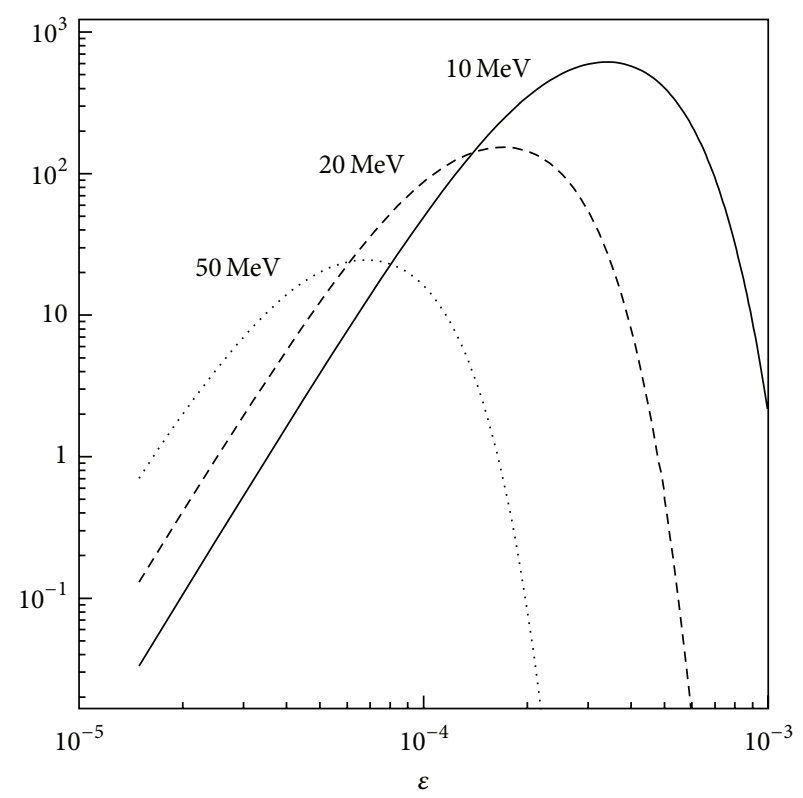

FIGURE 5: Number of dark photons from the process $J / \psi \rightarrow \eta^{\prime} \gamma^{\prime}$ with decay paths larger than $1 \mathrm{~cm}$ and lower than $5 \mathrm{~cm}$, as a function of $\epsilon$ and for various values of $m_{\gamma^{\prime}}$. An integrated luminosity of $1 \mathrm{ab}^{-1}$ is considered.

the $J / \psi$ peak. Considering that $\mathrm{BR}_{J / \psi \rightarrow \eta^{\prime} \gamma} \simeq 5 \cdot 10^{-3}$ [44], one obtains $\sim 150$ events for $\epsilon=10^{-4}$ and $L_{\text {int }}=1 \mathrm{ab}^{-1}$; On the other hand, for this value of kinetic mixing, the $\gamma^{\prime}$ mean decay path is of the right order of magnitude only for a limited range of mass values. It is, for instance, 11.2, 2.8 , and $0.45 \mathrm{~cm}$ for $m_{\gamma^{\prime}}=10,20$, and $50 \mathrm{MeV}$, respectively. The effect of this is seen in Figure 5 where the number of $\gamma^{\prime}$ decays occurring at a distance between 1 and $5 \mathrm{~cm}$ from the interaction point is plotted as a function of $\epsilon$, for different values of $m_{\gamma^{\prime}}$. Differently from the $e^{+} e^{-} \gamma$ case, here, it is the effect of the lifetime being short to be dominant, at least for the kinetic mixing values of interest, because for higher dark photon masses and low enough $\epsilon$, almost all of the $\gamma^{\prime}$ survive for less than $1 \mathrm{~cm}$. Note, also, that some further reduction in the number of observable events must be considered due to geometrical acceptance considerations. Still, there remains a small region of the parameter space for which one can hope to observe a reasonable number of $\gamma^{\prime}$ decays within acceptance.

There is however a further benefit specific of $V \rightarrow \mathrm{Pe}^{+} e^{-}$ events; these processes can in fact be used also to measure on an event-by-event basis the actual dark photon decay path, provided that the final state meson decays into at least a pair of charged particles. In this case, the position of the latter particles determines the primary production vertex, while the $\gamma^{\prime}$ decay position is determined, as usual, by the $e^{+} e^{-}$one.

For instance, for $J / \psi \rightarrow \eta^{\prime} \gamma^{\prime}$ events, one can use the process $\eta^{\prime} \rightarrow \eta \pi^{+} \pi^{-}$, where the $\pi^{+} \pi^{-}$pair allows determining exactly the collision point, at the price of reducing the total amount of useful events by a factor $\sim 0.43$ [44]. Since we are interested in millimeter scale decay paths, the photon conversion background should not be a problem 
anymore. However, there is a physical background to be kept now into consideration, namely, the Dalitz decay of the $J \psi$, $J / \psi \rightarrow \eta^{\prime} e^{+} e^{-}$. Its branching ratio can be estimated to be approximately $\mathrm{BR}\left(J / \psi \rightarrow \eta^{\prime} e^{+} e^{-}\right) \sim \mathrm{BR}\left(J / \psi \rightarrow \eta^{\prime} \gamma\right) \times 0.01$, so that the process is $\sim 10^{6}$ times more frequent than the signal, if $\epsilon=10^{-4}$. However, in this case, the $e^{+} e^{-}$and $\pi^{+} \pi^{-}$vertices must coincide within the detector's resolution, $\sigma_{\text {res }}$. Therefore, a reduction of the background by a factor $10^{4}$ can be achieved by accepting only events with measured decay paths larger than $4 \sigma_{\text {res }}$. Moreover, background events are expected to have a broad $e^{+} e^{-}$invariant mass distribution, while $\gamma^{\prime}$ decays are a narrow resonance in such channel. Not considering form factor effects, the number of background events, $N_{B}$, in a window of $\delta m$ around $m_{e^{+} e^{-}}=m_{\gamma^{\prime}}$ is given approximately by [9]

$$
\begin{aligned}
N_{B}= & N_{J / \psi \rightarrow \eta^{\prime} e^{+} e^{-}} \times B\left(\eta^{\prime} \longrightarrow \eta \pi^{+} \pi^{-}\right) \times \frac{\delta m}{m_{\gamma^{\prime}}} \\
& \times \frac{1}{\ln \left(\left(m_{J / \psi}-m_{\eta^{\prime}}\right) / 2 m_{e}\right)} .
\end{aligned}
$$

Note that the $1 / m_{\gamma^{\prime}}$ dependence on (5) favours the observation of higher mass dark photons. On the other hand, since the decay path scales as $1 / m_{\gamma^{\prime}}^{2}$, the effect of a finite vertex resolution favours the observation of lower mass particles.

In a given experiment, therefore, two parameters should ideally be kept as small as possible, $\sigma_{\text {res }}$ and $\delta m$. Take, for instance, $\epsilon=10^{-4}, m_{\gamma^{\prime}}=50 \mathrm{MeV}$. According to (5), the number of background events in the interesting mass bin would in this case be $\sim 2 \times 10^{5}$ for $\delta_{m}=1 \mathrm{MeV}$. By applying the $4 \sigma_{\text {res }}$ cut discussed above, this number reduces to $\sim 20$. Therefore, for $\sigma_{\text {res }}=1(0.5) \mathrm{mm}$, the signal significance (i.e., the number of signal events divided by the square root of the background) would be $\sim 4(6)$. It is important to underline that in this case the dimensions of the beam pipe are to first order irrelevant, since we are dealing with relatively short decay lengths. On the other hand, it has also to be noted, that we are here assuming full detection efficiency which might reveal an overoptimistic assumption. As for the continuum events, only detailed studies based on realistic detector parameters can finally assess the potentials of the method.

\section{Conclusions}

Experimental searches for a new, light, neutral boson, the "dark photon" or $\gamma^{\prime}$, are being pursued in many laboratories in the world, using different detection techniques. If the $\gamma^{\prime}$ is light enough and if its couplings with SM particles are suppressed by a factor $\leq 10^{-3}$ with respect to those of the ordinary photon, it can acquire a relatively long lifetime. This fact can be exploited at $e^{+} e^{-}$colliders by searching a $\gamma^{\prime} \rightarrow e^{+} e^{-}$decay vertex well separated by the primary production one, in $e^{+} e^{-} \rightarrow \gamma^{\prime} \gamma$ events. The paper shows that new generation $e^{+} e^{-}$colliders have the potentials to fully exploit this technique and can explore effective couplings down to few times $10^{-5}$ and $\gamma^{\prime}$ masses in the range $10-100 \mathrm{MeV}$ approximately. In general, higher energy machines are favoured, because the higher Lorentz boost of the produced (light) bosons allows a better separation of the secondary vertices. It is also seen, however, that the implementation of this method to real facilities requires a proper design of the interaction region and a wise choice of the tracking detector. To our knowledge, unfortunately, the beam pipe dimensions of SuperBelle are far from being optimal under this respect. On the other hand, since all of the future high luminosity $\tau$-charm factories are still in a preliminary design study phase, it is conceivable that in this case the interaction region can be designed so as to maximise the sensitivity for the proposed $\gamma^{\prime}$ search technique. Such a machine can also provide a complementary search method by the observation of displaced $e^{+} e^{-}$vertices in fully reconstructed $J / \psi \rightarrow \eta^{\prime} \gamma^{\prime}$ events, therefore, enhancing the interest for the construction of such a facility.

\section{Conflict of Interests}

The author declares that there is no conflict of interests regarding the publication of this paper.

\section{Acnowledgments}

The author would like to thank S. Andreas and D. Babusci for help and useful discussions.

\section{References}

[1] N. Arkani-Hamed, D. P. Finkbeiner, T. R. Slatyer, and N. Weiner, "A theory of dark matter," Physical Review D, vol. 79, no. 1, Article ID 015014, 2009.

[2] M. Pospelov and A. Ritz, "Astrophysical signatures of secluded dark matter," Physics Letters B, vol. 671, no. 3, pp. 391-397, 2009.

[3] C. Boehm and P. Fayet, "Scalar dark matter candidates," Nuclear Physics B, vol. 683, no. 1-2, pp. 219-263, 2004.

[4] N. Borodatchenkova, D. Choudhury, and M. Drees, "Probing $\mathrm{MeV}$ dark matter at low-energy e $\mathrm{e}^{+} \mathrm{e}^{-}$colliders," Physical Review Letters, vol. 96, no. 14, Article ID 141802, 2006.

[5] M. Pospelov, "Secluded U(1) below the weak scale," Physical Review D, vol. 80, no. 9, Article ID 095002, 2009.

[6] P. Fayet, " $U$-boson production in $\mathrm{e}^{+} \mathrm{e}^{-}$annihilations, $\psi$ and $\gamma$ decays, and light dark matter," Physical Review D, vol. 75, no. 11, Article ID 115017, 2007.

[7] J. D. Bjorken, R. Essig, P. Schuster, and N. Toro, "New fixedtarget experiments to search for dark gauge forces," Physical Review D, vol. 80, no. 7, Article ID 075018, 2009.

[8] B. Batell, M. Pospelov, and A. Ritz, "Probing a secluded U(1) at B factories," Physical Review D, vol. 79, no. 11, Article ID 115008, 2009.

[9] M. Reece and L. T. Wang, "Searching for the light dark gauge boson in GeV-scale experiments," Journal of High Energy Physics, vol. 0907, p. 051, 2009.

[10] B. Batell, M. Pospelov, and A. Ritz, "Exploring portals to a hidden sector through fixed targets," Physical Review D, vol. 80, no. 9, Article ID 095024, 2009.

[11] R. Essig, P. Schuster, and N. Toro, "Probing dark forces and light hidden sectors at low-energy $\mathrm{e}^{+} \mathrm{e}^{-}$colliders," Physical Review D, vol. 80, no. 1, Article ID 015003, 2009. 
[12] L. Barzè, G. Balossini, C. Bignamini et al., "Radiative events as a probe of dark forces at $\mathrm{GeV}$-scale $\mathrm{e}^{+} \mathrm{e}^{-}$colliders ," The European Physical Journal C, vol. 71, p. 1680, 2011.

[13] H. Merkel, P. Achenbach, C. A. Gayoso et al., "Search for light Gauge Boson of the dark sector at the mainz microtron," Physical Review Letters, vol. 106, no. 25, Article ID 251802, 2011.

[14] S. Abrahamyan, Z. Ahmed, K. Allada et al., "Search for a new Gauge Boson in electron-nucleus fixed-target scattering by the APEX experiment," Physical Review Letters, vol. 107, no. 19, Article ID 191804, 2011.

[15] J. P. Lees, V. Poireau, V. Tisserand et al., "Search for low-mass dark-sector Higgs Bosons," Physical Review Letters, vol. 108, no. 21, Article ID 211801, 2012.

[16] B. Echenard, "Search for low-mass dark matter at BABAR," Modern Physics Letters A, vol. 27, no. 18, Article ID 1230016, 2012.

[17] F. Archilli, D. Babusci, D. Badoni et al., "Search for a vector gauge boson in $\phi$ meson decays with the KLOE detector," Physics Letters B, vol. 706, no. 4-5, pp. 251-255, 2012.

[18] D. Babusci, D. Badoni, I. Balwierz-Pytko et al., "Limit on the production of a light vector gauge boson in $\phi$ meson decays with the KLOE detector," Physics Letters B, vol. 720, no. 1-3, pp. 111115, 2013.

[19] P. Adlarson, W. Augustyniak, W. Bardan et al., "Search for a dark photon in the $\pi^{0} \rightarrow e^{+} e^{-} \gamma$ decay," Physics Letters $B$, vol. 726, no. 1-3, pp. 187-193, 2013.

[20] S. Andreas, C. Niebuhr, and A. Ringwald, "New limits on hidden photons from past electron beam dumps," Physical Review D, vol. 86, no. 9, Article ID 095019, 2012.

[21] J. Blumlein and J. Brunner, "New exclusion limits for dark gauge forces from beam-dump data ," Physics Letters B, vol. 701, no. 2, pp. 155-159, 2011.

[22] S. N. Gninienko, "Stringent limits on the $\pi^{0} \rightarrow \gamma X, X \rightarrow e^{+} e^{-}$ decay from neutrino experiments and constraints on new light gauge bosons ," Physical Review D, vol. 85, no. 5, Article ID 055027, 2012.

[23] S. N. Gninienko, "Constraints on sub-GeV hidden sector gauge bosons from a search for heavy neutrino decays," Physics Letters B, vol. 713, no. 3, pp. 244-248, 2012.

[24] R. Essig, P. Schuster, N. Toro, and B. Wojtsekhwski, "An electron fixed target experiment to search for a new vector Boson A' Decaying to $\mathrm{e}^{+} \mathrm{e}^{-}$," Journal of High Energy Physics, vol. 1102, p. 009, 2011.

[25] P. Hansson Adrian et al., [HPS Collaboration], https://confluence.slac.stanford.edu/display/hpsg/HPS+Proposals.

[26] T. Beranek, H. Merkel, and M. Vanderhaeghen, "Theoretical framework to analyze searches for hidden light gauge bosons in electron scattering fixed target experiments," Physical Review $D$, vol. 88, Article ID 015032, 2013.

[27] M. Freytsis, G. Ovanesyan, and J. Thaler, "Dark force detection in low energy e-p collisions," Journal of High Energy Physics, vol. 2010, p. 111, 2010.

[28] B. Wojtsekhowski, D. Nikolenko, and I. Racheck, "Searching for a new force at VEPP-3," 2012, http://arxiv.org/abs/1207.5089.

[29] S. N. Gninienko, "Search for MeV dark photons in a lightshining-through-walls experiment at CERN," http://arxiv.org/ abs/1308.6521.

[30] D. Alesini, D. Babusci, M. E. Biagini et al., "DaØne upgrade for Siddharta run," LNF-06/33(IR), 2006.

[31] M. E. Biagini, S. Bini, R. Boni et al., "Tau/Charm factory accelerator report,” Tech. Rep. INFN-13-13(CLAB), 2013.
[32] Y. Ohnishi, T. Abe, T. Adachi et al., "Accelerator design at Super KEKB," Progress of Theoretical and Experimental Physics, vol. 2013, Article ID 03A011, 2013.

[33] P. Fayet, "Extra U(1)'s and new forces," Nuclear Physics B, vol. 347, no. 3, pp. 743-768, 1990.

[34] B. Holdom, "Two U(1)'s and $\in$ charge shifts," Physics Letters B, vol. 166, no. 2, pp. 196-198, 1986.

[35] Y. Kahn and J. Thalerm, "Searching for an invisible A' vector boson with DarkLight," Physical Review D, vol. 86, no. 11, Article ID 115012, 2012.

[36] P. de Niverville, M. Pospelov, and A. Ritz, "Observing a light dark matter beam with neutrino experiments," Physical Review $D$, vol. 84, no. 7, Article ID 075020, 2011.

[37] E. Izaguirre, G. Krnjaic, P. Schuster, and N. Toro, "New electron beam-dump experiments to search for $\mathrm{MeV}$ to few- $\mathrm{GeV}$ dark matter," Physical Review D, vol. 88, no. 11, Article ID 114015, 2013.

[38] R. Essig, J. Mardon, M. Papucci, T. Volansky, and Y.-M. Zhong, "Constraining light dark matter with low-energy e $\mathrm{e}^{+} \mathrm{e}^{-}$colliders," 2013, http://arxiv.org/abs/1309.5084.

[39] S. Andreas, "Light weakly interacting particles: constraints and connection to dark matter," DESY Report DESY-THESIS-2013024, 2013.

[40] H. K. Dreiner, J. F. Fortin, C. Hanhart, and L. Ubaldi, "Supernova constraints on $\mathrm{MeV}$ dark sectors from $\mathrm{e}^{+} \mathrm{e}^{-}$annihilations," http://arxiv.org/abs/1310.3826.

[41] D. Babusci, G. Bencivenni, C. Bloise et al., "KLOE," LNF10/17(P), 2010.

[42] F. Archilli, D. Badoni, F. Gonnella et al., “Technical design report of the inner tracker for the KLOE-2 experiment," Tech. Rep. LNF-10/3(P), 2010.

[43] P. Coyle and O. Schneider, "High spatial resolution detectors and particle lifetime measurements at LEP," Comptes Rendus Physique, vol. 3, no. 9, pp. 1143-1154, 2002.

[44] J. Beringer, J.-F. Arguin, R. M. Barnett et al., "Review of particle physics," Physical Review D, vol. 86, no. 1, Article ID 010001, 2012.

[45] J. Fu, H. B. Li, X. Qin, and M. Z. Yang, "Study of the electromagnetic transitions $J / \psi \rightarrow P I^{+} I^{-}$and probe dark photon," Modern Physics Letters A, vol. 27, no. 38, Article ID 1250223, 2012. 

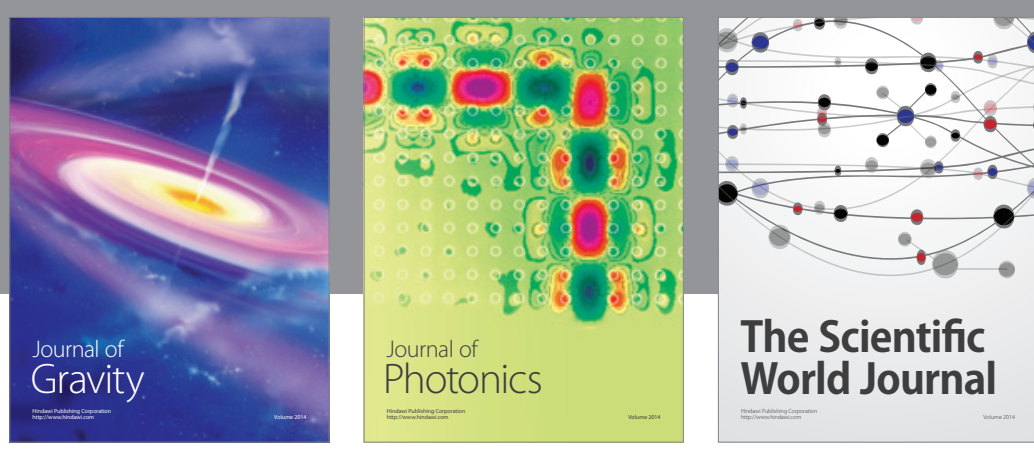

The Scientific World Journal
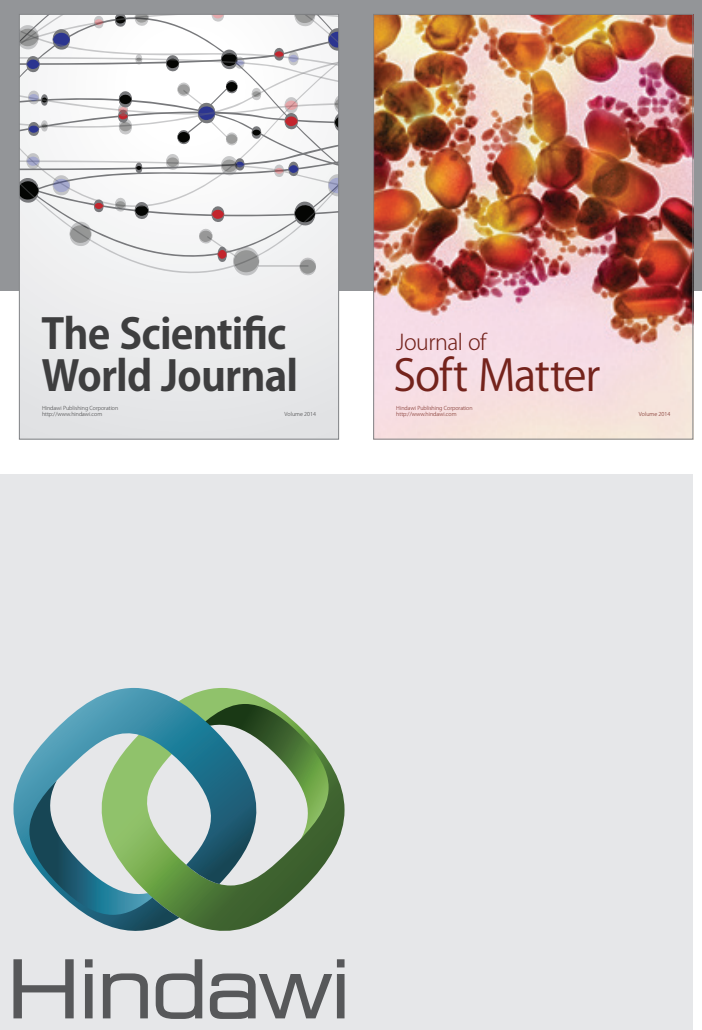

Submit your manuscripts at

http://www.hindawi.com

nternational Journal of

Statistical Mechanics
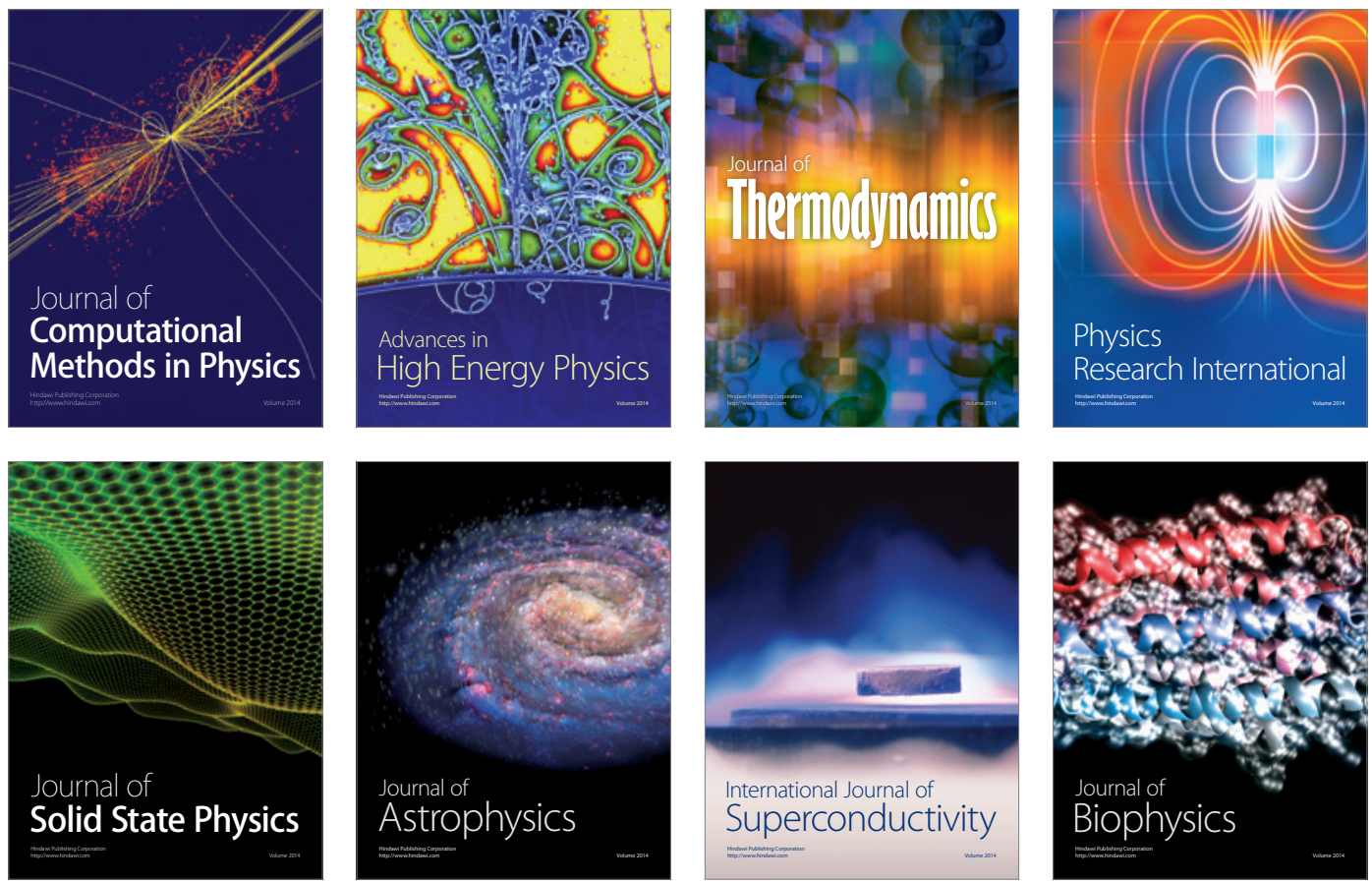
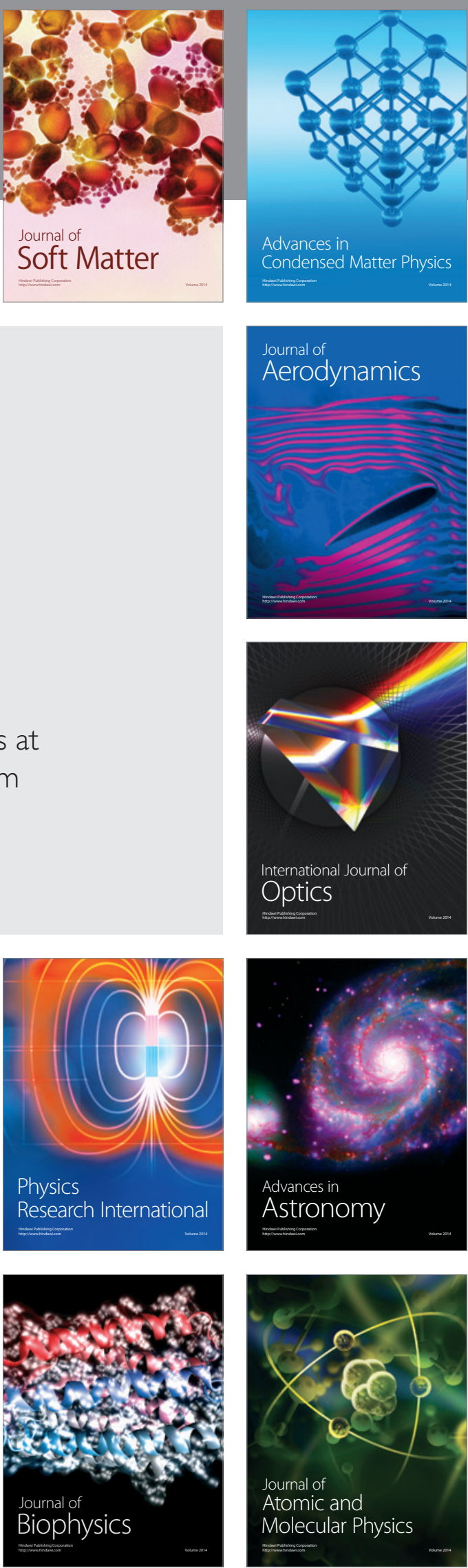